MATHEMATICS OF COMPUTATION

Volume 75, Number 255, July 2006, Pages 1449-1466

S 0025-5718(06)01836-9

Article electronically published on March 10, 2006

\title{
COMPUTING THE EHRHART QUASI-POLYNOMIAL OF A RATIONAL SIMPLEX
}

\author{
ALEXANDER BARVINOK
}

\begin{abstract}
We present a polynomial time algorithm to compute any fixed number of the highest coefficients of the Ehrhart quasi-polynomial of a rational simplex. Previously such algorithms were known for integer simplices and for rational polytopes of a fixed dimension. The algorithm is based on the formula relating the $k$ th coefficient of the Ehrhart quasi-polynomial of a rational polytope to volumes of sections of the polytope by affine lattice subspaces parallel to $k$-dimensional faces of the polytope. We discuss possible extensions and open questions.
\end{abstract}

\section{INTRODUCTION AND MAIN RESULTS}

Let $P \subset \mathbb{R}^{d}$ be a rational polytope, that is, the convex hull of a finite set of points with rational coordinates. Let $t \in \mathbb{N}$ be a positive integer such that the vertices of the dilated polytope

$$
t P=\{t x: x \in P\}
$$

are integer vectors. As is known (see, for example, Section 4.6 of [27]), there exist functions $e_{i}(P ; \cdot): \mathbb{N} \longrightarrow \mathbb{Q}, i=0, \ldots, d$, such that

$$
e_{i}(P ; n+t)=e_{i}(P ; n) \quad \text { for all } n \in \mathbb{N}
$$

and

$$
\left|n P \cap \mathbb{Z}^{d}\right|=\sum_{i=0}^{d} e_{i}(P ; n) n^{i} \quad \text { for all } n \in \mathbb{N} .
$$

The function on the right-hand side is called the Ehrhart quasi-polynomial of $P$. It is clear that if $\operatorname{dim} P=d$, then $e_{d}(P ; n)=\operatorname{vol} P$. In this paper, we are interested in the computational complexity of the coefficients $e_{i}(P ; n)$.

If the dimension $d$ is fixed in advance, the values of $e_{i}(P ; n)$ for any given $P, n$, and $i$ can be computed in polynomial time by interpolation, as implied by a polynomial time algorithm to count integer points in a polyhedron of a fixed dimension [4, [6].

If the dimension $d$ is allowed to vary, it is an NP-hard problem to check whether $P \cap \mathbb{Z}^{d} \neq \emptyset$, let alone to count integer points in $P$. This is true even when $P$ is a rational simplex, as exemplified by the knapsack problem; see, for example, Section 16.6 of [25]. If the polytope $P$ is integral, then the coefficients $e_{i}(P ; n)=e_{i}(P)$

Received by the editor April 29, 2005.

2000 Mathematics Subject Classification. Primary 52C07; Secondary 05A15, 68R05.

Key words and phrases. Ehrhart quasi-polynomial, rational polytope, valuation, algorithm.

This research was partially supported by NSF Grant DMS 0400617. 
do not depend on $n$. In that case, for any $k$ fixed in advance, computation of the Ehrhart coefficient $e_{d-k}(P)$ reduces in polynomial time to computation of the volumes of the $(d-k)$-dimensional faces of $P$ [5]. The algorithm is based on efficient formulas relating $e_{d-k}(P)$, volumes of the $(d-k)$-dimensional faces, and cones of feasible directions at those faces; see [22, [6], and [23. In particular, if $P=\Delta$ is an integer simplex, there is a polynomial time algorithm for computing $e_{d-k}(\Delta)$ as long as $k$ is fixed in advance.

In this paper, we extend the last result to rational simplices (a $d$-dimensional rational simplex is the convex hull in $\mathbb{R}^{d}$ of $(d+1)$ affinely independent points with rational coordinates).

- Let us fix an integer $k \geq 0$. The paper presents a polynomial time algorithm, which, given an integer $d \geq \bar{k}$, a rational simplex $\Delta \subset \mathbb{R}^{d}$, and a positive integer $n$, computes the value of $e_{d-k}(\Delta ; n)$.

We present the algorithm in Section 7 and discuss its possible extensions in Section 8 .

This is in contrast to the case of an integral polytope, for a general rational polytope $P$ computation of $e_{i}(P ; n)$ cannot be reduced to computation of the volumes of faces and some functionals of the "angles" (cones of feasible direction) at the faces. A general result of McMullen [19] (see also 21] and [20]) asserts that the contribution of the $i$-dimensional face $F$ of a rational polytope $P$ to the coefficient $e_{i}(P ; n)$ is a function of the volume of $F$, the cone of feasible directions of $P$ at $F$, and the translation class of the affine hull aff $(F)$ of $F$ modulo $\mathbb{Z}^{d}$.

Our algorithm is based on a new structural result, Theorem 1.1 below, relating the coefficient $e_{d-k}(P ; n)$ to volumes of sections of $P$ by affine lattice subspaces parallel to faces $F$ of $P$ with $\operatorname{dim} F \geq d-k$. Theorem 1.1 may be of interest in its own right.

1.1. Valuations and polytopes. Let $V$ be a $d$-dimensional real vector space and let $\Lambda \subset V$ be a lattice, that is, a discrete additive subgroup which spans $V$. A polytope $P \subset V$ is called a $\Lambda$-polytope or a lattice polytope if the vertices of $P$ belong to $\Lambda$. A polytope $P \subset V$ is called $\Lambda$-rational or just rational if $t P$ is a lattice polytope for some positive integer $t$.

For a set $A \subset V$, let $[A]: V \longrightarrow \mathbb{R}$ be the indicator of $A$ :

$$
[A](x)= \begin{cases}1 & \text { if } x \in A, \\ 0 & \text { if } x \notin A .\end{cases}
$$

A complex-valued function $\nu$ on rational polytopes $P \subset V$ is called a valuation if it preserves linear relations among indicators of rational polytopes:

$$
\sum_{i \in I} \alpha_{i}\left[P_{i}\right]=0 \Longrightarrow \sum_{i \in I} \alpha_{i} \nu\left(P_{i}\right)=0,
$$

where $P_{i} \subset V$ is a finite family of rational polytopes and $\alpha_{i}$ are rational numbers. We consider only $\Lambda$-valuations or lattice valuations $\nu$ that satisfy

$$
\nu(P+u)=\nu(P) \quad \text { for all } u \in \Lambda \text {; }
$$

see [21] and [20].

A general result of McMullen [19] states that if $\nu$ is a lattice valuation, $P \subset V$ is a rational polytope, and $t \in \mathbb{N}$ is a number such that $t P$ is a lattice polytope, then 
there exist functions $\nu_{i}(P ; \cdot): \mathbb{N} \longrightarrow \mathbb{C}, i=0, \ldots, d$, such that

$$
\nu(n P)=\sum_{i=0}^{d} \nu_{i}(P ; n) n^{i} \quad \text { for all } n \in \mathbb{N}
$$

and

$$
\nu_{i}(P ; n+t)=\nu_{i}(P ; n) \quad \text { for all } n \in \mathbb{N} .
$$

Clearly, if we compute $\nu(m P)$ for $m=n, n+t, \ldots, n+t d$, we can obtain $\nu_{i}(P ; n)$ by interpolation.

We are interested in the counting valuation $E$, where $V=\mathbb{R}^{d}, \Lambda=\mathbb{Z}^{d}$, and

$$
E(P)=\left|P \cap \mathbb{Z}^{d}\right|
$$

is the number of lattice points in $P$.

The idea of the algorithm is to replace valuation $E$ by some other valuation, so that the coefficients $e_{d}(P ; n), \ldots, e_{d-k}(P ; n)$ remain intact, but the new valuation can be computed in polynomial time on any given rational simplex $\Delta$, so that the desired coefficient $e_{d-k}(\Delta ; n)$ can be obtained by interpolation.

1.2. Valuations $E_{L}$. Let $L \subset \mathbb{R}^{d}$ be a lattice subspace, that is, a subspace spanned by the points $L \cap \mathbb{Z}^{d}$. Suppose that $\operatorname{dim} L=k$ and let $p r: \mathbb{R}^{d} \longrightarrow L$ be the orthogonal projection onto $L$. Let $P \subset \mathbb{R}^{d}$ be a rational polytope, let $Q=\operatorname{pr}(P)$, $Q \subset L$, be its projection, and let $\Lambda=\operatorname{pr}\left(\mathbb{Z}^{d}\right)$. Since $L$ is a lattice subspace, $\Lambda \subset L$ is a lattice.

Let $L^{\perp}$ be the orthogonal complement of $L$. Then $L^{\perp} \subset \mathbb{R}^{d}$ is a lattice subspace. We introduce the volume form $\operatorname{vol}_{d-k}$ on $L^{\perp}$ which differs from the volume form inherited from $\mathbb{R}^{d}$ by a scaling factor chosen so that the determinant of the lattice $\mathbb{Z}^{d} \cap L^{\perp}$ is 1 . Consequently, the same volume form $\operatorname{vol}_{d-k}$ is carried by all translations $x+L^{\perp}, x \in \mathbb{R}^{d}$.

We consider the following quantity

$$
E_{L}(P)=\sum_{m \in \Lambda} \operatorname{vol}_{d-k}\left(P \cap\left(m+L^{\perp}\right)\right)=\sum_{m \in Q \cap \Lambda} \operatorname{vol}_{d-k}\left(P \cap\left(m+L^{\perp}\right)\right)
$$

(clearly, for $m \notin Q$ the corresponding terms are 0 ).

In words, we take all lattice translates of $L^{\perp}$, select those that intersect $P$, and add the volumes of the intersections.

Clearly, $E_{L}$ is a lattice valuation, so

$$
E_{L}(n P)=\sum_{i=0}^{d} e_{i}(P, L ; n) n^{i}
$$

for some periodic functions $e_{i}(P, L ; \cdot)$. If $t P$ is an integer polytope for some $t \in \mathbb{N}$, then

$$
e_{i}(P, L ; n+t)=e_{i}(P, L ; n) \quad \text { for all } n \in \mathbb{N}
$$

and $i=0, \ldots, d$.

Note that if $L=\{0\}$, then $E_{L}(P)=\operatorname{vol} P$ and if $L=\mathbb{R}^{d}$, then $E_{L}(P)=\left|P \cap \mathbb{Z}^{d}\right|$, so the valuations $E_{L}$ interpolate between the volume and the number of lattice points as $\operatorname{dim} L$ grows.

We prove that $e_{d-k}(P ; n)$ can be represented as a linear combination of $e_{d-k}(P, L ; n)$ for some lattice subspaces $L$ with $\operatorname{dim} L \leq k$. 
Theorem 1.1. Let us fix an integer $k \geq 0$. Let $P \subset \mathbb{R}^{d}$ be a full-dimensional rational polytope and let $t$ be a positive integer such that $t P$ is an integer polytope. For $a(d-k)$-dimensional face $F$ of $P$ let $\operatorname{lin}(F) \subset \mathbb{R}^{d}$ be the $(d-k)$-dimensional subspace parallel to the affine hull aff $(F)$ of $F$ and let $L^{F}=(\operatorname{lin} F)^{\perp}$ be its orthogonal complement, so $L^{F} \subset \mathbb{R}^{d}$ is a $k$-dimensional lattice subspace.

Let $\mathcal{L}$ be a finite collection of lattice subspaces which contains the subspaces $L^{F}$ for all $(d-k)$-dimensional faces $F$ of $P$ and is closed under intersections. For $L \in \mathcal{L}$ let $\mu(L)$ be integer numbers such that the identity

$$
\left[\bigcup_{L \in \mathcal{L}} L\right]=\sum_{L \in \mathcal{L}} \mu(L)[L]
$$

holds for the indicator functions of the subspaces from $\mathcal{L}$.

Let us define

$$
\nu(n P)=\sum_{L \in \mathcal{L}} \mu(L) E_{L}(n P) \quad \text { for } n \in \mathbb{N} .
$$

Then there exist functions $\nu_{i}(P ; \cdot): \mathbb{N} \longrightarrow \mathbb{Q}, i=0, \ldots, d$, such that

$$
\nu(n P)=\sum_{i=0}^{d} \nu_{i}(P ; n) n^{i} \quad \text { for all } n \in \mathbb{N}
$$

$$
\nu_{i}(P ; n+t)=\nu_{i}(P ; n) \quad \text { for all } n \in \mathbb{N},
$$

$$
e_{d-i}(P ; n)=\nu_{d-i}(P ; n) \quad \text { for all } n \in \mathbb{N} \quad \text { and } \quad i=0, \ldots, k .
$$

We prove Theorem 1.1 in Section 4 after some preparations in Sections 2 and 3.

Remark 1.2. Valuation $E$ clearly does not depend on the choice of the scalar product in $\mathbb{R}^{d}$. One can observe that valuation $\nu$ of Theorem 1.1 admits a dual description which does not depend on the scalar product. Instead of $\mathcal{L}$, we consider the set $\mathcal{L}^{\vee}$ of subspaces containing the subspaces $\operatorname{lin}(F)$ and closed under taking sums of subspaces, and for $L \in \mathcal{L}^{\vee}$ we define $E_{L}^{\vee}(\cdot)$ as the sum of the volumes of sections of the polytope by the lattice affine subspaces parallel to $L$. Then

$$
\nu=\sum_{L \in \mathcal{L}^{\vee}} \mu^{\vee}(L) E_{L}^{\vee},
$$

where $\mu^{\vee}$ are some integers computed from the set $\mathcal{L}^{\vee}$, partially ordered by inclusion.

However, using the explicit scalar product turns out to be more convenient.

The advantage of working with valuations $E_{L}$ is that they are more amenable to computations.

- Let us fix an integer $k \geq 0$. We present a polynomial time algorithm, which, given an integer $d \geq k$, a $d$-dimensional rational simplex $\Delta \subset \mathbb{R}^{d}$, and a lattice subspace $L \subset \mathbb{R}^{d}$ such that $\operatorname{dim} L \leq k$, computes $E_{L}(\Delta)$.

We present the algorithm in Section 6 after some preparations in Section 5 . 
COMPUTING THE EHRHART QUASI-POLYNOMIAL OF A RATIONAL SIMPLEX 1453

1.3. The main ingredient of the algorithm to compute $e_{d-k}(\Delta ; n)$. Theorem 1.1 allows us to reduce the computation of $e_{d-k}(\Delta ; n)$ to that of $E_{L}(\Delta)$, where $L \subset \mathbb{R}^{d}$ is a lattice subspace and $\operatorname{dim} L \leq k$. Let us choose a particular lattice subspace $L$ with $\operatorname{dim} L=j \leq k$.

If $P=\Delta$ is a simplex, then the description of the orthogonal projection $Q=$ $\operatorname{pr}(\Delta)$ of $\Delta$ onto $L$ can be computed in polynomial time. Moreover, one can compute in polynomial time a decomposition of $Q$ into a union of non-intersecting polyhedral pieces $Q_{i}$, such that $\operatorname{vol}_{d-j}\left(p r^{-1}(x)\right)$ is a polynomial on each piece $Q_{i}$. Thus computing of $E_{L}(\Delta)$ reduces to computing of the sum

$$
\sum_{m \in Q_{i} \cap \Lambda} \phi(m)
$$

where $\phi$ is a polynomial with $\operatorname{deg} \phi=d-j, Q_{i} \subset L$ is a polytope with $\operatorname{dim} Q_{i}=$ $j \leq k$, and $\Lambda \subset L$ is a lattice. The sum is computed by applying the technique of "short rational functions" for lattice points in polytopes of a fixed dimension; cf. [7], 6], and [12.

The algorithm for computing the sum of a polynomial over integer points in a polytope is discussed in Section 5 .

\section{The Fourier expansions of $E$ and $E_{L}$}

Let $V$ be a $d$-dimensional real vector space with the scalar product $\langle\cdot, \cdot\rangle$ and the corresponding Euclidean norm $\|\cdot\|$. Let $\Lambda \subset V$ be a lattice and let $\Lambda^{*} \subset V$ be the dual or the reciprocal lattice

$$
\Lambda^{*}=\{x \in V:\langle x, y\rangle \in \mathbb{Z} \text { for all } y \in \Lambda\} .
$$

For $\tau>0$, we introduce the theta function

$$
\begin{aligned}
\theta_{\Lambda}(x, \tau) & =\tau^{d / 2} \sum_{m \in \Lambda} \exp \left\{-\pi \tau\|x-m\|^{2}\right\} \\
& =(\operatorname{det} \Lambda)^{-1} \sum_{l \in \Lambda^{*}} \exp \left\{-\pi\|l\|^{2} / \tau+2 \pi i\langle l, x\rangle\right\}, \quad \text { where } x \in V .
\end{aligned}
$$

The last inequality is the reciprocity relation for theta series (essentially, the Poisson summation formula); see, for example, Section 69 of 9 .

For a polytope $P$, let int $P$ denote the relative interior of $P$ and let $\partial P=P \backslash$ int $P$ be the boundary of $P$.

Lemma 2.1. Let $P \subset V$ be a full-dimensional polytope such that $\partial P \cap \Lambda=\emptyset$. Then

$$
\begin{aligned}
|P \cap \Lambda| & =\lim _{\tau \longrightarrow+\infty} \int_{P} \theta_{\Lambda}(x, \tau) d x \\
& =(\operatorname{det} \Lambda)^{-1} \lim _{\tau \longrightarrow+\infty} \sum_{l \in \Lambda^{*}} \exp \left\{-\pi\|l\|^{2} / \tau\right\} \int_{P} \exp \{2 \pi i\langle l, x\rangle\} d x .
\end{aligned}
$$

Proof. As is known (cf., for example, Section B.5 of [17]), as $\tau \longrightarrow+\infty$, the function $\theta_{\Lambda}(x, \tau)$ converges in the sense of distributions to the sum of the delta-functions concentrated at the points $m \in \Lambda$. Therefore, for every smooth function $\phi: \mathbb{R}^{d} \longrightarrow$ $\mathbb{R}$ with a compact support, we have

$$
\lim _{\tau \longrightarrow+\infty} \int_{\mathbb{R}^{d}} \phi(x) \theta_{\Lambda}(x, \tau) d x=\sum_{m \in \Lambda} \phi(m) .
$$


Since $\partial P \cap \Lambda=\emptyset$, we can replace $\phi$ by the indicator function $[P]$ in (2.1).

Remark 2.2. If $\partial P \cap \Lambda \neq \emptyset$, the limit still exists but then it counts every lattice point $m \in \partial P$ with the weight equal to the "solid angle" of $m$ at $P$, since every term $\exp \left\{-\pi \tau\|x-m\|^{2}\right\}$ is spherically symmetric about $m$. This connection between the solid angle valuation and the theta function was described by the author in the unpublished paper 2] (the paper is very different from paper [5] which has the same title) and independently discovered by Diaz and Robins [13. Diaz and Robins used a similar approach based on Fourier analysis to express coefficients of the Ehrhart polynomial of an integer polytope in terms of cotangent sums [14. Banaszczyk [1] obtained asymptotically optimal bounds in transference theorems for lattices by using a similar approach with theta functions, with the polytope $P$ replaced by a Euclidean ball.

The formula of Lemma 2.1 can be considered as the Fourier expansion of the counting valuation.

We need a similar result for valuation $E_{L}$ defined in Section 1.2.

Lemma 2.3. Let $P \subset \mathbb{R}^{d}$ be a full-dimensional polytope and let $L \subset \mathbb{R}^{d}$ be a lattice subspace with $\operatorname{dim} L=k$. Let $p r: \mathbb{R}^{d} \longrightarrow L$ be the orthogonal projection onto $L$, let $Q=\operatorname{pr}(P)$, and let $\Lambda=\operatorname{pr}\left(\mathbb{Z}^{d}\right)$, so $\Lambda \subset L$ is a lattice in $L$. Suppose that $\partial Q \cap \Lambda=\emptyset$.

Then

$$
E_{L}(P)=\lim _{\tau \longrightarrow+\infty} \sum_{l \in L \cap \mathbb{Z}^{d}} \exp \left\{-\pi\|l\|^{2} / \tau\right\} \int_{P} \exp \{2 \pi i\langle l, x\rangle\} d x
$$

Proof. We observe that $L \cap \mathbb{Z}^{d}=\Lambda^{*}$. For a vector $x \in \mathbb{R}^{d}$, let $x_{L}$ be the orthogonal projection of $x$ onto $L$. Applying the reciprocity relation for theta functions in $L$, we write

$$
\begin{aligned}
& \sum_{l \in L \cap \mathbb{Z}^{d}} \exp \left\{-\pi\|l\|^{2} / \tau+2 \pi i\langle l, x\rangle\right\} \\
= & \sum_{l \in L \cap \mathbb{Z}^{d}} \exp \left\{-\pi\|l\|^{2} / \tau+2 \pi i\left\langle l, x_{L}\right\rangle\right\} \\
= & (\operatorname{det} \Lambda) \tau^{k / 2} \sum_{m \in \Lambda} \exp \left\{-\pi \tau\left\|x_{L}-m\right\|^{2}\right\} .
\end{aligned}
$$

As is known (cf., for example, Section B.5 of [17]), as $\tau \longrightarrow+\infty$, the function

$$
g_{\tau}(x)=\tau^{k / 2} \sum_{m \in \Lambda} \exp \left\{-\pi \tau\left\|x_{L}-m\right\|^{2}\right\}
$$

converges in the sense of distributions to the sum of the delta-functions concentrated on the subspaces $m+L^{\perp}$ (this is the set of points where $x_{L}=m$ ) for $m \in \Lambda$.

Therefore, for every smooth function $\phi: \mathbb{R}^{d} \longrightarrow \mathbb{R}$ with a compact support, we have

$$
\lim _{\tau \longrightarrow+\infty} \int_{\mathbb{R}^{d}} \phi(x) g_{\tau}(x) d x=\sum_{m \in \Lambda} \int_{m+L^{\perp}} \phi(x) d_{L^{\perp}} x,
$$

where $d_{L^{\perp}} x$ is the Lebesgue measure on $m+L^{\perp}$ induced from $\mathbb{R}^{d}$. 
Since $\partial Q \cap \Lambda=\emptyset$, each subspace $m+L^{\perp}$ for $m \in \Lambda$ either intersects the interior of $P$ or is at least some distance $\epsilon=\epsilon(P, L)>0$ away from $P$. Hence we may replace $\phi$ by the indicator $[P]$ in $(2.2)$.

Recall from Section 1.2 that measuring volumes in $m+L^{\perp}$, we scale the volume form in $L^{\perp}$ induced from $\mathbb{R}^{d}$ so that the determinant of the lattice $L^{\perp} \cap \mathbb{Z}^{d}$ is 1 . One can observe that $\operatorname{det} \Lambda$ provides the required normalization factor, so

$$
(\operatorname{det} \Lambda) \int_{m+L^{\perp}}[P](x) d_{L^{\perp}}(x)=\operatorname{vol}_{d-k}\left(P \cap\left(m+L^{\perp}\right)\right) .
$$

The proof now follows.

Remark 2.4. If $\partial Q \cap \Lambda \neq \emptyset$, the limit still exists, but then for $m \in \partial Q \cap \Lambda$ the volume $\operatorname{vol}_{d-k}\left(P \cap\left(m+L^{\perp}\right)\right)$ is counted with the weight defined as follows: we find the minimal (under inclusion) face $F$ of $P$ such that $m+L^{\perp}$ is contained in $\operatorname{aff}(F)$ and the weight is equal to the solid angle of $P$ at $F$.

\section{Exponential VALUATions}

Let $V$ be a $d$-dimensional Euclidean space, let $\Lambda \subset V$ be a lattice, and let $\Lambda^{*}$ be the reciprocal lattice. Let us choose a vector $l \in \Lambda^{*}$ and let us consider the integral

$$
\Phi_{l}(P)=\int_{P} \exp \{2 \pi i\langle l, x\rangle\} d x
$$

where $d x$ is the Lebesgue measure in $V$. Note that for $l=0$ we have $\Phi_{l}(P)=$ $\Phi_{0}(P)=\operatorname{vol} P$. We have

$$
\Phi_{l}(P+a)=\exp \{2 \pi i\langle l, a\rangle\} \Phi_{l}(P) \quad \text { for all } a \in V .
$$

It follows that $\Phi_{l}$ is a $\Lambda$-valuation on rational polytopes $P \subset V$.

If $l \neq 0$, then the following lemma (essentially, Stokes' formula) shows that $\Phi_{l}$ can be expressed as a linear combination of exponential valuations on the facets of $P$. The proof can be found, for example, in $[3$.

Lemma 3.1. Let $P \subset V$ be a full-dimensional polytope. For a facet $\Gamma$ of $P$, let $d_{\Gamma} x$ be the Lebesgue measure on $\operatorname{aff}(\Gamma)$, and let $p_{\Gamma}$ be the unit outer normal to $\Gamma$. Then, for every $l \in V \backslash 0$, we have

$$
\int_{P} \exp \{2 \pi i\langle l, x\rangle\} d x=\sum_{\Gamma} \frac{\left\langle l, p_{\Gamma}\right\rangle}{2 \pi i\|l\|^{2}} \int_{\Gamma} \exp \{2 \pi i\langle l, x\rangle\} d_{\Gamma} x
$$

where the sum is taken over all facets $\Gamma$ of $P$.

Let $F \subset P$ be an $i$-dimensional face of $P$. Recall that by $\operatorname{lin}(F)$ we denote the $i$-dimensional subspace of $\mathbb{R}^{d}$ that is parallel to the affine hull aff $(F)$ of $F$. We need the following result.

Theorem 3.2. Let $P \subset V$ be a rational full-dimensional polytope and let $t$ be a positive integer such that $t P$ is a lattice polytope. Let $\epsilon \geq 0$ be a rational number and let $a \in V$ be a vector. Let us choose $l \in \Lambda^{*}$. Then there exist functions $f_{i}(P, \epsilon, a, l ; \cdot): \mathbb{N} \longrightarrow \mathbb{C}, i=0, \ldots, d$, such that

$(1)$

$$
\Phi_{l}((n+\epsilon) P+a)=\sum_{i=0}^{d} f_{i}(P, \epsilon, a, l ; n) n^{i} \quad \text { for all } n \in \mathbb{N}
$$

and 
$(2)$

$$
\begin{aligned}
& \quad f_{i}(P, \epsilon, a, l ; n+t)=f_{i}(P, \epsilon, a, l ; n) \text { for all } n \in \mathbb{N} \\
& \text { and } i=0, \ldots, d .
\end{aligned}
$$

Suppose that $f_{d-k}(P, \epsilon, a, l ; n) \neq 0$ for some $n$. Then there exists $a(d-k)$ dimensional face $F$ of $P$ such that $l$ is orthogonal to $\operatorname{lin}(F)$.

Proof. Since

$$
\Phi_{l}(P+a)=\exp \{2 \pi i\langle l, a\rangle\} \Phi_{l}(P),
$$

without loss of generality we assume that $a=0$. We will denote $f_{i}(P, \epsilon, 0, l ; n)$ just by $f_{i}(P, \epsilon, l ; n)$.

We proceed by induction on $d$. For $d=0$ the statement of the theorem obviously holds. Suppose that $d \geq 1$. If $l=0$, then $\Phi_{l}((n+\epsilon) P)=(n+\epsilon)^{d} \operatorname{vol} P$ and the statement holds as well.

Suppose that $l \neq 0$. For a facet $\Gamma$ of $P$, let $\Lambda_{\Gamma}=\Lambda \cap \operatorname{lin}(\Gamma)$ and let $l_{\Gamma}$ be the orthogonal projection of $l$ onto $\operatorname{lin}(\Gamma)$. Thus $\Lambda_{\Gamma}$ is a lattice in the $(d-1)$-dimensional Euclidean space $\operatorname{lin}(\Gamma)$ and $l_{\Gamma} \in \Lambda_{\Gamma}^{*}$, so we can define valuations $\Phi_{l_{\Gamma}}$ on $\operatorname{lin}(\Gamma)$. Since $t P$ is a lattice polytope, for every facet $\Gamma$ there is a vector $u_{\Gamma} \in V$ such that

$$
\operatorname{lin}(\Gamma)=\operatorname{aff}(t \Gamma)-t u_{\Gamma} \quad \text { and } \quad t u_{\Gamma} \in \Lambda .
$$

Let $\Gamma^{\prime}=\Gamma-u_{\Gamma}$, so $\Gamma^{\prime} \subset \operatorname{lin}(\Gamma)$ is a $\Lambda_{\Gamma}$-rational $(d-1)$-dimensional polytope such that $t \Gamma^{\prime}$ is a $\Lambda_{\Gamma}$-polytope. We have

$$
(n+\epsilon) \Gamma=(n+\epsilon) \Gamma^{\prime}+(n+\epsilon) u_{\Gamma} .
$$

Applying Lemma 3.1 to $(n+\epsilon) P$, we get

$$
\Phi_{l}((n+\epsilon) P)=\sum_{\Gamma} \psi(\Gamma, l ; n) \Phi_{l_{\Gamma}}\left((n+\epsilon) \Gamma^{\prime}\right),
$$

where

$$
\psi(\Gamma, l ; n)=\frac{\left\langle l, p_{\Gamma}\right\rangle}{2 \pi i\|l\|^{2}} \exp \left\{2 \pi i(n+\epsilon)\left\langle l, u_{\Gamma}\right\rangle\right\}
$$

and the sum is taken over all facets $\Gamma$ of $P$.

Since $t u_{\Gamma} \in \Lambda$ and $l \in \Lambda^{*}$, we have

$$
\psi(\Gamma, l ; n+t)=\psi(\Gamma, l ; n) \quad \text { for all } n \in \mathbb{N} .
$$

Hence, applying the induction hypothesis, we may write

$$
f_{i}(P, \epsilon, l ; n)=\sum_{\Gamma} \psi(\Gamma, l ; n) f_{i}\left(\Gamma^{\prime}, \epsilon, l_{\Gamma} ; n\right) \quad \text { for all } n \in \mathbb{N}
$$

and $i=0, \ldots, d-1$ and $f_{d}(P, \epsilon, l ; n) \equiv 0$. Hence (1)-(2) follows by the induction hypothesis.

If $f_{d-k}(P, \epsilon, l ; n) \neq 0$, then there is a facet $\Gamma$ of $P$ such that $f_{d-k}\left(\Gamma^{\prime}, \epsilon, l_{\Gamma} ; n\right) \neq 0$. By the induction hypothesis, there is a face $F^{\prime}$ of $\Gamma^{\prime}$ such that $\operatorname{dim} F^{\prime}=d-k$, and $l_{\Gamma}$ is orthogonal to $\operatorname{lin}\left(F^{\prime}\right)$. Then $F=F^{\prime}+u_{\Gamma}$ is a $(d-k)$-dimensional face of $P$, $\operatorname{lin}\left(F^{\prime}\right)=\operatorname{lin}(F)$, and $l$ is orthogonal to $\operatorname{lin}(F)$, which completes the proof. 


\section{Proof of Theorem 1.1}

First, we discuss some ideas relevant to the proof.

4.1. Shifting a valuation by a polytope. Let $V$ be a $d$-dimensional real vector space, let $\Lambda \subset V$ be a lattice, and let $\nu$ be a $\Lambda$-valuation on rational polytopes. Let us fix a rational polytope $R \subset V$. McMullen [19] observed that the function $\mu$ defined by

$$
\mu(P)=\nu(P+R)
$$

is a $\Lambda$-valuation on rational polytopes $P$. Here "+" stands for the Minkowski sum:

$$
P+R=\{x+y: x \in P, y \in R\} .
$$

This result follows since the transformation $P \longmapsto P+R$ preserves linear dependencies among indicators of polyhedra; cf. [21.

Let $t$ be a positive integer such that $t P$ is a lattice polytope. McMullen [19. deduced that there exist functions $\nu_{i}(P, R ; \cdot): \mathbb{N} \longrightarrow \mathbb{C}, i=0, \ldots, d$, such that

$$
\nu(n P+R)=\sum_{i=0}^{d} \nu_{i}(P, R ; n) n^{i} \quad \text { for all } n \in \mathbb{N}
$$

and

$$
\nu_{i}(P, R ; n+t)=\nu_{i}(P, R ; n) \text { for all } n \in \mathbb{N} .
$$

4.2. Continuity properties of valuations $E$ and $E_{L}$. Let $R \subset \mathbb{R}^{d}$ be a fulldimensional rational polytope containing the origin in its interior. Then for every polytope $P \subset \mathbb{R}^{d}$ and every $\epsilon>0$ we have $P \subset(P+\epsilon R)$. We observe that

$$
\left|(P+\epsilon R) \cap \mathbb{Z}^{d}\right|=\left|P \cap \mathbb{Z}^{d}\right|
$$

for all sufficiently small $\epsilon>0$. If $P$ is a rational polytope, the supporting affine hyperplanes of the facets of $n P$ for $n \in \mathbb{N}$ are split among finitely many translation classes modulo $\mathbb{Z}^{d}$. Therefore, there exists $\delta=\delta(P, R)>0$ such that

$$
\left|(n P+\epsilon R) \cap \mathbb{Z}^{d}\right|=\left|n P \cap \mathbb{Z}^{d}\right| \quad \text { for all } 0<\epsilon<\delta \quad \text { and all } n \in \mathbb{N} .
$$

We also note that for every rational subspace $L \subset \mathbb{R}^{d}$, we have

$$
\lim _{\epsilon \longrightarrow 0+} E_{L}(P+\epsilon R)=E_{L}(P) .
$$

We will use the perturbation $P \longmapsto P+\epsilon R$ to push valuations $E$ and $E_{L}$ into a sufficiently generic position, so that we can apply Lemmas 2.1 and 2.3 without having to deal with various boundary effects. This is somewhat similar in spirit to the idea of $[8]$.

4.3. Linear identities for quasi-polynomials. Let us fix positive integers $t$ and $d$. Suppose that we have a possibly infinite family of quasi-polynomials $p_{l}: \mathbb{N} \longrightarrow \mathbb{C}$ of the type

$$
p_{l}(n)=\sum_{i=0}^{d} p_{i}(l ; n) n^{i} \quad \text { for all } n \in \mathbb{N},
$$

where functions $p_{i}(l ; \cdot): \mathbb{N} \longrightarrow \mathbb{C}, i=0, \ldots, d$, satisfy

$$
p_{i}(l ; n)=p_{i}(l ; n+t) \quad \text { for all } n \in \mathbb{N} .
$$


Suppose further that $p: \mathbb{N} \longrightarrow \mathbb{C}$ is yet another quasi-polynomial

$$
p(n)=\sum_{i=0}^{d} p_{i}(n) n^{i} \quad \text { where } p_{i}(n+t)=p_{i}(n) \quad \text { for all } n \in \mathbb{N} .
$$

Finally, suppose that $c_{l}(\cdot): \mathbb{R}_{+} \longrightarrow \mathbb{C}$ is a family of functions and that

$$
p(n)=\lim _{\tau \longrightarrow+\infty} \sum_{l} c_{l}(\tau) p_{l}(n) \quad \text { for all } n \in \mathbb{N}
$$

and that the series converges absolutely for every $n \in \mathbb{N}$ and every $\tau>0$.

Then we claim that for $i=0, \ldots, d$ we have

$$
p_{i}(n)=\lim _{\tau \longrightarrow+\infty} \sum_{l} c_{l}(\tau) p_{i}(l ; n) \quad \text { for all } n \in \mathbb{N}
$$

and that the series converges absolutely for every $n \in \mathbb{N}$ and every $\tau>0$.

This follows since $p_{i}(n)$, respectively $p_{i}(l ; n)$, can be expressed as linear combinations of $p(m)$, respectively $p_{l}(m)$, for $m=n, n+t, \ldots, n+t d$ with the coefficients depending on $m, n, t$, and $d$ only.

Now we are ready to prove Theorem 1.1.

4.4. Proof of Theorem 1.1. Let us fix a rational polytope $P \subset \mathbb{R}^{d}$ as defined in the statement of the theorem. For $L \in \mathcal{L}$ let $P_{L} \subset L$ be the orthogonal projection of $P$ onto $L$ and let $\Lambda_{L} \subset L$ be the orthogonal projection of $\mathbb{Z}^{d}$ onto $L$.

Let $a \in \operatorname{int} P$ be a rational vector and let

$$
R=P-a .
$$

Hence $R$ is a rational polytope containing the origin in its interior. Let $R_{L}$ denote the orthogonal projection of $R$ onto $L$.

Since $P$ is a rational polytope and $\mathcal{L}$ is a finite set of rational subspaces, there exists $\delta=\delta(P, R)>0$ such that for all $0<\epsilon<\delta$ and all $n \in \mathbb{N}$, we have

$$
(n P+\epsilon R) \cap \mathbb{Z}^{d}=n P \cap \mathbb{Z}^{d} \quad \text { and } \quad \partial(n P+\epsilon R) \cap \mathbb{Z}^{d}=\emptyset \quad \text { for all } n \in \mathbb{N}
$$

and for all $L \in \mathcal{L}$, we have

$$
\begin{aligned}
& \left(n P_{L}+\epsilon R_{L}\right) \cap \Lambda_{L}=n P_{L} \cap \Lambda_{L} \quad \text { and } \\
& \partial\left(n P_{L}+\epsilon R_{L}\right) \cap \Lambda_{L}=\emptyset \quad \text { for all } n \in \mathbb{N} ;
\end{aligned}
$$

cf. Section 4.2. Let us choose any rational $0<\epsilon<\delta$.

Because of (4.1), we can write

$$
\left|(n P+\epsilon R) \cap \mathbb{Z}^{d}\right|=\sum_{i=0}^{d} e_{i}(P ; n) n^{i} \quad \text { for all } n \in \mathbb{N}
$$

and by Lemma 2.1 we get

$$
\left|(n P+\epsilon R) \cap \mathbb{Z}^{d}\right|=\lim _{\tau \longrightarrow+\infty} \sum_{l \in \mathbb{Z}^{d}} \exp \left\{-\pi\|l\|^{2} / \tau\right\} \Phi_{l}(n P+\epsilon R),
$$

where $\Phi_{l}$ are the exponential valuations of Section 3 .

Since $\Phi_{l}$ is a $\mathbb{Z}^{d}$-valuation, by Section 4.1 there exist functions $f_{i}(P, \epsilon, l ; \cdot): \mathbb{N} \longrightarrow$ $\mathbb{C}, i=0, \ldots, d$, such that

$$
\Phi_{l}(n P+\epsilon R)=\sum_{i=0}^{d} f_{i}(P, \epsilon, l ; n) n^{i} \quad \text { for } n \in \mathbb{N}
$$


and

$$
f_{i}(P, \epsilon, l ; n+t)=f_{i}(P, \epsilon, l ; n) \text { for all } n \in \mathbb{N} .
$$

Moreover, we can write

$$
n P+\epsilon R=n P+\epsilon(P-a)=(n+\epsilon) P-\epsilon a .
$$

Therefore, by Theorem 3.2, for $i \leq k$ we have $f_{d-i}(P, \epsilon, l ; n)=0$ unless $l \in L^{F}$ for some face $F$ of $P$ with $\operatorname{dim} F=d-k$. Therefore, combining (4.3)-(4.6) and Section 4.3, we obtain for all $0 \leq i \leq k$ and all $n \in \mathbb{N}$

$$
\begin{aligned}
e_{d-i}(P ; n) & =\lim _{\tau \longrightarrow+\infty} \sum_{l \in \mathbb{Z}^{d}} \exp \left\{-\pi\|l\|^{2} / \tau\right\} f_{d-i}(P, \epsilon, l ; n) \\
& =\lim _{\tau \longrightarrow+\infty} \sum_{l \in \bigcup_{L \in \mathcal{L}}\left(L \cap \mathbb{Z}^{d}\right)} \exp \left\{-\pi\|l\|^{2} / \tau\right\} f_{d-i}(P, \epsilon, l ; n),
\end{aligned}
$$

since vectors $l \in \mathbb{Z}^{d}$ outside of subspaces $L \in \mathcal{L}$ contribute 0 to the sum. Therefore, for $0 \leq i \leq k$ and all $n \in \mathbb{N}$

$$
e_{d-i}(P ; n)=\lim _{\tau \longrightarrow+\infty} \sum_{L \in \mathcal{L}} \mu(L) \sum_{l \in L \cap \mathbb{Z}^{d}} \exp \left\{-\pi\|l\|^{2} / \tau\right\} f_{d-i}(P, \epsilon, l ; n)
$$

On the other hand, because of (4.2), by Lemma 2.3 we get for all $L \in \mathcal{L}$ and all $n \in \mathbb{N}$

$$
E_{L}(n P+\epsilon R)=\lim _{\tau \longrightarrow+\infty} \sum_{l \in L \cap \mathbb{Z}^{d}} \exp \left\{-\pi\|l\|^{2} / \tau\right\} \Phi_{l}(n P+\epsilon R)
$$

Since $E_{L}$ are $\mathbb{Z}^{d}$-valuations, by Section 4.1 there exist functions $e_{i}(P, \epsilon, L ; \cdot): \mathbb{N} \longrightarrow \mathbb{Q}, i=0, \ldots, d$, such that

$$
E_{L}(n P+\epsilon R)=\sum_{i=0}^{d} e_{i}(P, \epsilon, L ; n) n^{i} \quad \text { for all } n \in \mathbb{N}
$$

and

$$
e_{i}(P, \epsilon, L ; n+t)=e_{i}(P, \epsilon, L ; n) \quad \text { for all } n \in \mathbb{N} .
$$

Combining (4.5)-(4.6) and (4.8)-(4.10), by Section 4.3 we conclude

$$
e_{d-i}(P, \epsilon, L ; n)=\lim _{\tau \longrightarrow+\infty} \sum_{l \in L \cap \mathbb{Z}^{d}} \exp \left\{-\pi\|l\|^{2} / \tau\right\} f_{d-i}(P, \epsilon, l ; n) \quad \text { for all } n \in \mathbb{N} \text {. }
$$

Therefore, by (4.7), for $0 \leq i \leq k$ we have

$$
e_{d-i}(P ; n)=\sum_{L \in \mathcal{L}} \mu(L) e_{d-i}(P, \epsilon, L ; n) \quad \text { for all } n \in \mathbb{N} .
$$

Since $E_{L}$ is a $\mathbb{Z}^{d}$-valuation, there exist functions $e_{i}(P, L ; \cdot): \mathbb{N} \longrightarrow \mathbb{Q}, i=$ $0, \ldots, d$, such that

$$
E_{L}(n P)=\sum_{i=0}^{d} e_{i}(P, L ; n) n^{i} \quad \text { for all } n \in \mathbb{N}
$$

and

$$
e_{i}(P, L ; n+t)=e_{i}(P, L ; n) \quad \text { for all } n \in \mathbb{N} .
$$

Let us choose an $m \in \mathbb{N}$. Substituting $n=m, m+t, \ldots, m+t d$ in (4.12), we obtain $e_{i}(P, L ; m)$ as a linear combination of $E_{L}(n P)$ with coefficients depending on $n, m$, 
$t$, and $d$ only. Similarly, substituting $n=m, m+t, \ldots, m+t d$ in (4.9), we obtain $e_{i}(P, \epsilon, L ; m)$ as the same linear combination of $E_{L}(n P+\epsilon R)$. Since volumes are continuous functions, in view of (4.2) (see also Section 4.2), we get

$$
\lim _{\epsilon \rightarrow 0+} E_{L}(n P+\epsilon R)=E_{L}(n P) \quad \text { for } n=m, m+t, \ldots, m+t d .
$$

Therefore,

$$
\lim _{\epsilon \longrightarrow 0+} e_{i}(P, \epsilon, L ; m)=e_{i}(P, L ; m) \quad \text { for all } m \in \mathbb{N} .
$$

Taking the limit as $\epsilon \longrightarrow 0+$ in (4.11), we obtain for $0 \leq i \leq k$

$$
e_{d-i}(P ; n)=\sum_{L \in \mathcal{L}} \mu(L) e_{d-i}(P, L ; n) \quad \text { for all } n \in \mathbb{N} .
$$

To complete the proof, we note that

$$
\nu_{d-i}(P, L ; n)=\sum_{L \in \mathcal{L}} \mu(L) e_{d-i}(P, L ; n) .
$$

\section{Summing UP A POLYNOMial OVER INTEGer POINTS IN A RATIONAL POLYTOPE}

Let us fix a positive integer $k$ and let us consider the following situation. Let $Q \subset \mathbb{R}^{k}$ be a rational polytope, let int $Q$ be the relative interior of $Q$, and let $f: \mathbb{R}^{k} \longrightarrow \mathbb{R}$ be a polynomial with rational coefficients. We want to compute the value

$$
\sum_{m \in \operatorname{int} Q \cap \mathbb{Z}^{k}} f(m) .
$$

We claim that as soon as the dimension $k$ of the polytope $Q$ is fixed, there is a polynomial time algorithm to do that. We assume that the polytope $Q$ is given by the list of its vertices and the polynomial $f$ is given by the list of its coefficients.

For an integer point $m=\left(\mu_{1}, \ldots, \mu_{k}\right)$, let

$$
\mathbf{x}^{m}=x_{1}^{\mu_{1}} \cdots x_{k}^{\mu_{k}} \quad \text { for } \mathbf{x}=\left(x_{1}, \ldots, x_{k}\right)
$$

be the Laurent monomial in $k$ variables $\mathbf{x}=\left(x_{1}, \ldots, x_{k}\right)$. We use the following result [6].

5.1. The short rational function algorithm. Let us fix $k$. There is a polynomial time algorithm, which, given a rational polytope $Q \subset \mathbb{R}^{k}$, computes the generating function (Laurent polynomial)

$$
S(Q ; \mathbf{x})=\sum_{m \in \operatorname{int} Q \cap \mathbb{Z}^{k}} \mathbf{x}^{m}
$$

in the form

$$
S(Q ; \mathbf{x})=\sum_{i \in I} \epsilon_{i} \frac{\mathbf{x}^{a_{i}}}{\left(1-\mathbf{x}^{b_{i 1}}\right) \cdots\left(1-\mathbf{x}^{b_{i k}}\right)},
$$

where $a_{i} \in \mathbb{Z}^{k}, b_{i j} \in \mathbb{Z}^{k} \backslash\{0\}$, and $\epsilon_{i} \in \mathbb{Q}$. In particular, the number $|I|$ of fractions is bounded by a polynomial in the input size of $Q$.

Our first step is computing the generating function

$$
S(Q, f ; \mathbf{x})=\sum_{m \in \operatorname{int} Q \cap \mathbb{Z}^{k}} f(m) \mathbf{x}^{m} .
$$


Our approach is similar to that of [12, although we obtain better complexity bounds (our algorithm is polynomial in $\operatorname{deg} f$ whereas the algorithm of [12] is exponential in $\operatorname{deg} f)$.

5.2. The algorithm for computing $S(Q, f ; \mathbf{x})$. We observe that

$$
S(Q, f ; \mathbf{x})=f\left(x_{1} \frac{\partial}{\partial x_{1}}, \ldots, x_{k} \frac{\partial}{\partial x_{k}}\right) S(Q ; \mathbf{x}) .
$$

We compute $S(Q ; \mathbf{x})$ as in Section 5.1.

Let $a=\left(\alpha_{1}, \ldots, \alpha_{k}\right)$ be an integer vector, let $b_{j}=\left(\beta_{j 1}, \ldots, \beta_{j k}\right)$ be non-zero integer vectors for $j=1, \ldots, k$, and let $\gamma_{1}, \ldots, \gamma_{k}$ be positive integers. Then

$$
\begin{aligned}
& \left(x_{i} \frac{\partial}{\partial x_{i}}\right) \frac{\mathbf{x}^{a}}{\left(1-\mathbf{x}^{b_{1}}\right)^{\gamma_{1}} \cdots\left(1-\mathbf{x}^{b_{k}}\right)^{\gamma_{k}}} \\
= & \alpha_{i} \frac{\mathbf{x}^{a}}{\left(1-\mathbf{x}^{b_{1}}\right)^{\gamma_{1}} \cdots\left(1-\mathbf{x}^{b_{k}}\right)^{\gamma_{k}}}+\sum_{j=1}^{k} \gamma_{j} \beta_{j i} \frac{\mathbf{x}^{a+b_{j}}}{\left(1-\mathbf{x}^{b_{j}}\right)^{\gamma_{j}+1}} \prod_{s \neq j} \frac{1}{\left(1-\mathbf{x}^{b_{s}}\right)^{\gamma_{s}}} .
\end{aligned}
$$

Consecutively applying the above formula and collecting similar fractions, we compute

$$
f\left(x_{1} \frac{\partial}{\partial x_{1}}, \ldots, x_{k} \frac{\partial}{\partial x_{k}}\right) \frac{\mathbf{x}^{a}}{\left(1-\mathbf{x}^{b_{1}}\right) \cdots\left(1-\mathbf{x}^{b_{k}}\right)}
$$

as an expression of the type

$$
\sum_{j} \rho_{j} \frac{\mathbf{x}^{a_{j}}}{\left(1-\mathbf{x}^{b_{1}}\right)^{\gamma_{j 1}} \cdots\left(1-\mathbf{x}^{b_{k}}\right)^{\gamma_{j k}}}
$$

where $\rho_{j} \in \mathbb{Q}, \gamma_{j 1}, \ldots, \gamma_{j k}$ are non-negative integers satisfying $\gamma_{j 1}+\cdots+\gamma_{j k} \leq$ $k+\operatorname{deg} f$ and $a_{j}$ are vectors of the type

$$
a_{j}=a+\mu_{1} b_{1}+\cdots+\mu_{k} b_{k}
$$

where $\mu_{i}$ are non-negative integers and $\mu_{1}+\cdots+\mu_{k} \leq \operatorname{deg} f$. The number of terms in (5.2) is bounded by $(\operatorname{deg} f)^{O(k)}$, which shows that for a $k$ fixed in advance, the algorithm runs in polynomial time.

Consequently, $S(Q, f ; \mathbf{x})$ is computed in polynomial time.

Formally speaking, to compute the sum (5.1), we have to substitute $x_{i}=1$ into the formula for $S(Q, f ; \mathbf{x})$. This, however, cannot be done in a straightforward way since $\mathbf{x}=(1, \ldots, 1)$ is a pole of every fraction in the expression for $S(Q, f ; \mathbf{x})$. Nevertheless, the substitution can be done via efficient computation of the relevant residue of $S(Q, f ; \mathbf{x})$ as described in [4] and [7].

5.3. The algorithm for computing the sum. The output of Algorithm 5.2 represents $S(Q, f ; \mathbf{x})$ in the general form

$$
S(Q, f ; \mathbf{x})=\sum_{i \in I} \epsilon_{i} \frac{\mathbf{x}^{a_{i}}}{\left(1-\mathbf{x}^{b_{i 1}}\right)^{\gamma_{i 1}} \cdots\left(1-\mathbf{x}^{b_{i k}}\right)^{\gamma_{i k}}},
$$

where $\epsilon_{i} \in \mathbb{Q}, a_{i} \in \mathbb{Z}^{k}, b_{i j} \in \mathbb{Z}^{k} \backslash\{0\}$, and $\gamma_{i j} \in \mathbb{N}$ are such that $\gamma_{i 1}+\cdots+\gamma_{i k} \leq$ $k+\operatorname{deg} f$ for all $i \in I$.

Let us choose a vector $l \in \mathbb{Q}^{k}, l=\left(\lambda_{1}, \ldots, \lambda_{k}\right)$, such that $\left\langle l, b_{i j}\right\rangle \neq 0$ for all $i, j$ (such a vector can be computed in polynomial time; cf. [4). For a complex $\tau$, let

$$
\mathbf{x}(\tau)=\left(e^{\tau \lambda_{1}}, \ldots, e^{\tau \lambda_{k}}\right) \text {. }
$$


We want to compute the limit

$$
\lim _{\tau \longrightarrow 0} G(\tau) \quad \text { for } G(\tau)=S(Q, f ; \mathbf{x}(\tau)) .
$$

In other words, we want to compute the constant term of the Laurent expansion of $G(\tau)$ around $\tau=0$.

Let us consider a typical fraction

$$
\frac{\mathbf{x}^{a}}{\left(1-\mathbf{x}^{b_{1}}\right)^{\gamma_{1}} \cdots\left(1-\mathbf{x}^{b_{k}}\right)^{\gamma_{k}}} .
$$

Substituting $\mathbf{x}(\tau)$, we get the expression

$$
\frac{e^{\alpha \tau}}{\left(1-e^{\tau \beta_{1}}\right)^{\gamma_{1}} \cdots\left(1-e^{\tau \beta_{k}}\right)^{\gamma_{k}}},
$$

where $\alpha=\langle a, l\rangle$ and $\beta_{i}=\left\langle b_{i}, l\right\rangle$ for $i=1, \ldots, k$. The order of the pole at $\tau=0$ is $D=\gamma_{1}+\cdots+\gamma_{k} \leq k+\operatorname{deg} f$. To compute the constant term of the Laurent expansion of (5.3) at $\tau=0$, we do the following.

We compute the polynomial

$$
q(\tau)=\sum_{i=0}^{D} \frac{\alpha^{i}}{i !} \tau^{i}
$$

that is the truncation at $\tau^{D}$ of the Taylor series expansion of $e^{\alpha \tau}$. For $i=1, \ldots, k$ we compute the polynomial $p_{i}(\tau)$ with $\operatorname{deg} p_{i}=D$ such that

$$
\frac{\tau}{1-e^{\tau \beta_{i}}}=p_{i}(\tau)+\text { terms of higher order in } \tau
$$

at $\tau=0$. Consecutively multiplying polynomials $\bmod \tau^{D+1}$, we compute a polynomial $u(\tau)$ with $\operatorname{deg} u=D$ such that

$$
q(\tau) p_{1}^{\gamma_{1}}(\tau) \cdots p_{k}^{\gamma_{k}}(\tau) \equiv u(\tau) \quad \bmod \tau^{D+1} .
$$

The coefficient of $\tau^{D}$ in $u(\tau)$ is the desired constant term of the Laurent expansion.

\section{Computing $E_{L}(\Delta)$}

Let us fix a positive integer $k$. Let $\Delta \subset \mathbb{R}^{d}$ be a rational simplex given by the list of its vertices and let $L \subset \mathbb{R}^{d}$ be a rational subspace given by its basis and such that $\operatorname{dim} L=k$. In this section, we describe a polynomial time algorithm for computing the value of $E_{L}(\Delta)$ as defined in Section 1.2.

Let $p r: \mathbb{R}^{d} \longrightarrow L$ be the orthogonal projection. We compute the vertices of the polytope $Q=\operatorname{pr}(\Delta)$ and a basis of the lattice $\Lambda=\operatorname{pr}\left(\mathbb{Z}^{d}\right)$. For basic lattice algorithms see 25] and [16].

As is known, as $x \in \Delta$ varies, the function

$$
\phi(x)=\operatorname{vol}_{d-k}\left(P_{x}\right) \quad \text { where } P_{x}=\left(\Delta \cap\left(x+L^{\perp}\right)\right)
$$

is a piecewise polynomial on $Q$. Our first step consists of computing a decomposition

$$
Q=\bigcup_{i} C_{i}
$$

such that $C_{i} \subset Q$ are rational polytopes (chambers) with pairwise disjoint interiors and polynomials $\phi_{i}: L \longrightarrow \mathbb{R}$ such that $\phi_{i}(x)=\phi(x)$ for $x \in C_{i}$.

We observe that every vertex of $P_{x}$ is the intersection of $x+L^{\perp}$ and some $k$ dimensional face $F$ of $\Delta$. 
For every face $G$ of $\Delta$ with $\operatorname{dim} G=k-1$ and such that $\operatorname{aff}(G)$ is not parallel to $L^{\perp}$, let us compute

$$
A_{G}=\left\{x \in L: x+L^{\perp} \cap \operatorname{aff}(G) \neq \emptyset\right\} .
$$

Then $A_{G}$ is an affine hyperplane in $L$. The number of different hyperplanes $A_{G}$ is $d^{O(k)}$ and hence they cut $Q$ into at most $d^{O\left(k^{2}\right)}$ polyhedral chambers $C_{i}$; cf. Section 6.1 of 18 . As long as $x$ stays within the relative interior of a chamber $C_{i}$, the strong combinatorial type of $P_{x}$ does not change (the facets of $P_{x}$ move parallel to themselves) and hence the restriction $\phi_{i}$ of $\phi$ onto $C_{i}$ is a polynomial; cf. Section 5.1 of [24]. Since in the $(d-k)$-dimensional space $x+L^{\perp}$ the polytope $P_{x}$ is defined by $d$ linear inequalities, $\phi_{i}$ can be computed in polynomial time; see [15] and [3].

The decomposition (6.1) gives rise to the formula

$$
[Q]=\sum_{j}\left[Q_{j}\right]
$$

where $Q_{j}$ are open faces of the chambers $C_{i}$ (the number of such faces is bounded by a polynomial in $d$ ); cf. Section 6.1 of [18. Hence we have

$$
E_{L}(\Delta)=\sum_{j} \sum_{m \in Q_{j} \cap \Lambda} \phi(m)
$$

Each inner sum is the sum of a polynomial over lattice points in a polytope of dimension at most $k$. By a change of the coordinates, it becomes the sum over integer points in a rational polytope and we compute it as described in Section 5.

\section{Computing $e_{d-k}(\Delta ; n)$}

Let us fix an integer $k \geq 0$. We describe our algorithm, which, given a positive integer $d \geq k$, a rational simplex $\Delta \subset \mathbb{R}^{d}$ (defined, for example, by the list of its vertices), and a positive integer $n$, computes the number $e_{d-k}(\Delta ; n)$.

We use Theorem 1.1.

7.1. Computing the set $\mathcal{L}$ of subspaces. We compute subspaces $L$ and numbers $\mu(L)$ described in Theorem 1.1. Namely, for each $(d-k)$-dimensional face $F$ of $\Delta$, we compute a basis of the subspace $L^{F}=(\operatorname{lin} F)^{\perp}$. Hence $\operatorname{dim} L^{F} \leq k$. Clearly, the number of distinct subspaces $L^{F}$ is $d^{O(k)}$. We let $\mathcal{L}$ be the set consisting of the subspaces $L^{F}$ and all other subspaces obtained as intersections of $L^{F}$. We compute $\mathcal{L}$ in $k$ (or fewer) steps. Initially, we let

$$
\mathcal{L}:=\left\{L^{F}: F \text { is a }(d-k) \text {-dimensional face of } \Delta\right\} .
$$

Then, at every step, we consider the previously constructed subspaces $L \in \mathcal{L}$, consider the pairwise intersections $L \cap L^{F}$ as $F$ ranges over the $(d-k)$-dimensional faces of $\Delta$, and add the obtained subspace $L \cap L^{F}$ to the set $\mathcal{L}$ if it is not already there. If no new subspaces are obtained, we stop. Clearly, in the end of this process, we will obtain all subspaces $L$ that are intersections of different $L^{F_{i}}$. Since $\operatorname{dim} L^{F_{i}}=k$, each subspace $L \in \mathcal{L}$ is an intersection of some $k$ subspaces $L^{F_{i}}$. Hence the process stops after $k$ steps and the total number $|\mathcal{L}|$ of subspaces is $d^{O\left(k^{2}\right)}$.

Having computed the subspaces $L \in \mathcal{L}$, we compute the numbers $\mu(L)$ as follows. 
For each pair of subspaces $L_{1}, L_{2} \in \mathcal{L}$ such that $L_{1} \subset L_{2}$, we compute the number $\mu\left(L_{1}, L_{2}\right)$ recursively: if $L_{1}=L_{2}$, we let $\mu\left(L_{1}, L_{2}\right)=1$. Otherwise, we let

$$
\mu\left(L_{1}, L_{2}\right)=-\sum_{\substack{L \in \mathcal{L} \\ L_{1} \subset L \subset L_{2} \\ L \neq L_{2}}} \mu\left(L_{1}, L\right) .
$$

In the end, for each $L \in \mathcal{L}$, we let

$$
\mu(L)=\sum_{\substack{L_{1} \in \mathcal{L} \\ L \subset L_{1}}} \mu\left(L, L_{1}\right) .
$$

Hence $\mu\left(L_{i}, L_{j}\right)$ are the values of the Möbius function on the set $\mathcal{L}$ partially ordered by inclusion, so

$$
\left[\bigcup_{L \in \mathcal{L}} L\right]=\sum_{L \in \mathcal{L}} \mu(L)[L]
$$

follows from the Möbius inversion formula; cf. Section 3.7 of 27 .

Now, for each $L \in \mathcal{L}$ and $m=n, n+t, \ldots, n+t d$ we compute the values of $E_{L}(m \Delta)$ as in Section 6, compute

$$
\nu(m \Delta)=\sum_{L \in \mathcal{L}} \mu(L) E_{L}(m \Delta),
$$

and find $\nu_{d-k}(\Delta ; n)=e_{d-k}(\Delta, n)$ by interpolation.

\section{Possible extensions And Further QUestions}

8.1. Computing more general expressions. Let $P \subset \mathbb{R}^{d}$ be a rational polytope, let $\alpha \geq 0$ be a rational number, and let $u \in \mathbb{R}^{d}$ be a rational vector. One can show (cf. Section 4.1) that

$$
\left|((n+\alpha) P+u) \cap \mathbb{Z}^{d}\right|=\sum_{i=0}^{d} e_{i}(P, \alpha, u ; n) n^{i} \quad \text { for all } n \in \mathbb{N},
$$

where $e_{i}(P, \alpha, u ; \cdot): \mathbb{N} \longrightarrow \mathbb{Q}, i=0, \ldots, d$, satisfy

$$
e_{i}(P, \alpha, u ; n+t)=e_{i}(P, \alpha, u ; n) \text { for all } n \in \mathbb{N},
$$

provided $t \in \mathbb{N}$ is a number such that $t P$ is an integer polytope. As long as $k$ is fixed in advance, for given $\alpha, u, n$, and a rational simplex $\Delta \subset \mathbb{R}^{d}$, one can compute $e_{d-k}(\Delta, \alpha, u ; n)$ in polynomial time. Similarly, Theorem 1.1 and its proof extend to this more general situation in a straightforward way.

8.2. Computing the generating function. Let $P \subset \mathbb{R}^{d}$ be a rational polytope. Then, for every $0 \leq i \leq d$, the series

$$
\sum_{n=1}^{+\infty} e_{i}(P ; n) t^{n}
$$

converges to a rational function $f_{i}(P ; t)$ for $|t|<1$.

It is not clear whether $f_{d-k}(\Delta ; t)$ can be efficiently computed as a "closed form expression" in any meaningful sense, although it seems that by adjusting the methods of Sections 5-7, for any given $t$ such that $|t|<1$ one can compute the value of $f_{d-k}(\Delta ; t)$ in polynomial time (again, $k$ is assumed to be fixed in advance). 
8.3. Extensions to other classes of polytopes. If $k$ is fixed in advance, the coefficient $e_{d-k}(P ; n)$ can be computed in polynomial time, if the rational polytope $P \subset \mathbb{R}^{d}$ is given by the list of its $d+c$ vertices or the list of its $d+c$ inequalities, where $c$ is a constant fixed in advance. A similar result holds for rational parallelepipeds $P$, that is, for Minkowski sums of $d$ rational intervals that do not lie in the same affine hyperplane in $\mathbb{R}^{d}$.

8.4. Possible applications to integer programming and integer point counting. If $P \subset \mathbb{R}^{m}$ is a rational polytope given by the list of its defining linear inequalities, the problem of testing whether $P \cap \mathbb{Z}^{m}=\emptyset$ is a typical problem of integer programming; see [16] and [25. Moreover, a general construction of "aggregation" (see Section 16.6 of [25] and Section 2.2 of [26]) establishes a bijection between the sets $P \cap \mathbb{Z}^{m}$ and $\Delta \cap \mathbb{Z}^{d}$ provided $P$ is defined by $d+1$ linear inequalities. Here $\Delta \subset \mathbb{R}^{d}$ is a rational simplex whose definition is computable in polynomial time from that of $P$. It would be interesting to find out whether approximating valuation $E$ by valuation $\nu$ of Theorem 1.1 for some $k \ll d$ and applying the algorithm of this paper to compute $\nu(\Delta)$ can be of any practical use to solve higher-dimensional integer programs and integer point counting problems. It could complement existing software packages [11] and [10] based on the "short rational functions" calculus.

\section{REFERENCES}

1. W. Banaszczyk, New bounds in some transference theorems in the geometry of numbers, Math. Ann. 296 (1993), 625-635. MR.1233487 (94k:11075)

2. A. Barvinok, Computing the Ehrhart polynomial of a convex lattice polytope, preprint TRITAMAT-1992-0036 (1992), Royal Institute of Technology, Stockholm.

3. A. Barvinok, Computing the volume, counting integral points, and exponential sums, Discrete Comput. Geom. 10 (1993), 123-141. MR1220543(94d:52005)

4. A. Barvinok, A polynomial time algorithm for counting integral points in polyhedra when the dimension is fixed, Math. Oper. Res. 19 (1994), 769-779. MR.1304623 (96c:52026)

5. A. Barvinok, Computing the Ehrhart polynomial of a convex lattice polytope, Discrete Comput. Geom. 12 (1994), 35-48. MR.1280575(95e:52015)

6. A. Barvinok and J. Pommersheim, An algorithmic theory of lattice points in polyhedra, New perspectives in algebraic combinatorics (Berkeley, CA, 1996-97), Math. Sci. Res. Inst. Publ., vol. 38, Cambridge Univ. Press, Cambridge, 1999, pp. 91-147. MR1731815 (2000k:52014)

7. A. Barvinok and K. Woods, Short rational generating functions for lattice point problems, J. Amer. Math. Soc. 16 (2003), 957-979. MR1992831 (2004e:05009)

8. M. Beck and F. Sottile, Irrational proofs for three theorems of Stanley, preprint arXiv math.CO/0501359, 2005.

9. R. Bellman, A Brief Introduction to Theta Functions, Athena Series: Selected Topics in Mathematics, Holt Rinehart and Winston, New York, 1961. MR0125252 (23:A2556)

10. K. Beyls, M. Bruynooghe, V. Loechner, R. Seghir, and S. Verdoolaege Analytical computation of Ehrhart polynomials: Enabling more compiler analyses and optimizations, Proceedings of the 2004 International Conference on Compilers, Architecture, and Synthesis for Embedded Systems, ACM Press, New York, 2004 pp. 248-258; see also http:// www.kotnet.org $/ \sim_{\text {skimo/barvinok. }}$

11. J. A. De Loera, R. Hemmecke, J. Tauzer, and R. Yoshida, Effective lattice point counting in rational convex polytopes, J. Symbolic Comput. 38(2004), 1273-1302; see also http:// www.math.ucdavis.edu/ latte. MR2094541 (2005i:52020)

12. J. A. De Loera, R. Hemmecke, M. Köppe, and R. Weismantel, Integer polynomial optimization in fixed dimension, preprint, arXiv: math.OC/0410111, 2004.

13. R. Diaz and S. Robins, Solid angles, lattice points, and the Fourier decomposition of polytopes, manuscript, 1994. 
14. R. Diaz and S. Robins, The Ehrhart polynomial of a lattice polytope, Ann. of Math. (2) 145 (1997), 503-518; Erratum: 146 (1997), no. 1, 237. MR.1454701 (98e:11117a) MR 1469320 $(98 \mathrm{e}: 11117 \mathrm{~b})$

15. P. Gritzmann and V. Klee, On the complexity of some basic problems in computational convexity. II. Volume and mixed volumes, Polytopes: Abstract, Convex and Computational (Scarborough, ON, 1993), NATO Adv. Sci. Inst. Ser. C. Math. Phys. Sci., vol. 440, Kluwer Acad. Publ., Dordrecht, 1994, pp. 373-466. MR.1322071 (96k:52012)

16. M. Grötschel, L. Lovász, and A. Schrijver, Geometric Algorithms and Combinatorial Optimization. Second edition, Algorithms and Combinatorics, vol. 2, Springer-Verlag, Berlin, 1993. MR.1261419 (95e:90001)

17. P. D. Lax, Functional Analysis, Pure and Applied Mathematics, Wiley-Interscience, New York, 2002. MR1892228 (2003a:47001)

18. J. Matoušek, Lectures on Discrete Geometry, Graduate Texts in Mathematics, vol. 212, Springer-Verlag, New York, 2002. MR.1899299 (2003f:52011)

19. P. McMullen, Lattice invariant valuations on rational polytopes, Arch. Math. (Basel) 31 1978/79, 509-516. MR0526617 (80d:52011)

20. P. McMullen, Valuations and dissections, Handbook of Convex Geometry, vol. B, NorthHolland, Amsterdam, 1993, pp. 933-988. MR1243000 (95f:52018)

21. P. McMullen and R. Schneider, Valuations on convex bodies, Convexity and its Applications, Birkhäuser, Basel, 1983, pp. 170-247. MR0731112 (85e:52001)

22. R. Morelli, Pick's theorem and the Todd class of a toric variety, Adv. Math. 100 (1993), 183-231. MR.1234309 (94j:14048)

23. J. Pommersheim and H. Thomas, Cycles representing the Todd class of a toric variety, J. Amer. Math. Soc. 17 (2004), 983-994. MR2083474 (2005h:14124)

24. R. Schneider, Convex Bodies: The Brunn-Minkowski Theory, Encyclopedia of Mathematics and its Applications, vol. 44, Cambridge University Press, Cambridge, 1993. MR1216521 (94d:52007)

25. A. Schrijver, Theory of Linear and Integer Programming, Wiley-Interscience Series in Discrete Mathematics, John Wiley \& Sons, Ltd., Chichester, 1986. MR0874114 (88m:90090)

26. V. N. Shevchenko, Qualitative Topics in Integer Linear Programming, Translated from the Russian by H. H. McFaden. Translations of Mathematical Monographs, vol. 156, American Mathematical Society, Providence, RI, 1997. MR.1414900 (97m:90001)

27. R. P. Stanley, Enumerative Combinatorics. Vol. 1, corrected reprint of the 1986 original. Cambridge Studies in Advanced Mathematics, vol. 49, Cambridge University Press, Cambridge, 1997. MR.1442260 (98a:05001)

Department of Mathematics, University of Michigan, Ann Arbor, Michigan 481091043

E-mail address: barvinok@umich.edu 